\title{
Influencia del tamaño del campo y horario del partido en la respuestafísica de equipos de la Segunda División Española de Fútbol Effect of pitch size and time of the match in the physical performance of teams the Spanish Second Division

\author{
*Jaime Gutierrez, **Julen Castellano, ***David Casamichana, *Javier Sánchez-Sanchez
} \\ *Universidad Pontificia de Salamanca (España), **Universidad del País Vasco (España), ***Universidad Europea del Atlántico (España)
}

Resumen. El objetivo del presente estudio ha sido analizar la influencia de las variables situacionales tamaño del campo y horario del partido sobre la distancia total recorrida por partido y los metros completados en diferentes rangos de velocidad de equipos de la Liga de Fútbol Profesional Española. Los 22 equipos de la $2^{\text {a }}$ División 'A' tomaron parte del estudio durante la temporada 2013/2014, dando como resultado 634 registros. Se estudió el comportamiento físico de los equipos por medio del registro de la distancia total recorrida y la completada en cinco rangos de velocidad: distancia recorrida a velocidad parado (DPa) $<2 \mathrm{~km} / \mathrm{h}$; distancia recorrida a velocidad caminando (DCa) 2-7.3 km/h; distancia recorrida a velocidad trotando (DTr) 7.3-14 km/h; distancia recorrida a velocidad corriendo (DCo) 14-21 km/h; distancia recorrida a velocidad esprintando (DEs) $\geq 21 \mathrm{~km} / \mathrm{h}$. Para ello se utilizó el sistema de seguimiento multicámara computarizado TRACAB (Chyronhego®, Estados Unidos). Los resultados mostraron que los equipos recorrieron más distancia ( $\mathrm{p}<0,05)$ en un campo grande (CG) que en uno pequeño (CP) (11.905 \pm 463 m vs. $11.722 \pm 631$ m, respectivamente). En el CG se realizaron más metros $(\mathrm{p}<0,01)$ en DPa y DCo, pero menos $(\mathrm{p}<0,05)$ en DCa que en el CP. El horario del partido sólo mostró diferencias en DCo, siendo mayor $(\mathrm{p}<0,05)$ en horario de tarde y noche, respecto al de mañana. Conocer la influencia de variables situacionales en la demanda física podría ser clave para planificar los ciclos de entrenamiento, establecer el plan estratégico pre-partido o afinar los protocolos de recuperación. Palabras Clave: Fútbol, variables situacionales, distancia total, rendimiento físico.

Abstract. The purpose of this study was to analyze the influence of the situational variables, pitch size and time of the match, on the total distance meters covered per match and distance covered at different speed zones per match of teams from the Professional Football League. 22 teams from the Professional football Spanish Second Division took part of the study competing during the season 2013/2014, totalling 634 registers. Physical performance of the teams was studied through total distance covered and meters covered at 5 different speed zones: distance covered standing (DPa) $<2 \mathrm{~km} / \mathrm{h}$; distance covered walking (Dca) 2-7.3 km/h; distance covered jogging (DTr) 7.3-14 km/h; distance covered running (DCo) 14-21 km/h; distance covered sprinting (DEs) $\geq 21 \mathrm{~km} / \mathrm{h}$. The multicamera computerized tracking system TRACAB (Chyronhego®, United States) was used. Results showed that teams covered greater distances $(\mathrm{p}<0,05)$ in a big pitch $(\mathrm{CG})$ than in a small one $(\mathrm{CP})(11.905 \pm 463 \mathrm{~m}$ vs. $11.722 \pm 631 \mathrm{~m}$, respectively). More meters were covered in CG $(p<0,01)$ than in DPa and DCo, but less $(p<0,05)$ in DCa than in CP. The time of the match caused the DCo to be greater $(\mathrm{p}<0,05)$ in the afternoon and night compared to the morning. Knowing the influence of situational variables on team's physical performance is essential to planning training cycles and establishing a strategic plan before each match.

Key Words: Football, situational variables, total distance, physical performance.

\section{Introducción}

El análisis de los patrones de movimiento durante los partidos de fútbol ha sido utilizado para evaluar las demandas físicas en esta disciplina desde los años 30 (Ryan, Bahnert, McBrien, Siegler \& Lovell, 2015). Sin embargo, no ha sido hasta hace poco tiempo cuando han aparecido sofisticados sistemas de monitorización(Castellano, ÁlvarezPastor \& Bradley, 2014), que han permitido extraer información que puede ser aplicada a la mejora de los procesos de entrenamiento de futbolista (Carling, Bloomfield, Nelsen \& Reilly, 2008). Los sistemas de monitorización actualmente utilizados permiten seguir a cada jugador en el campo durante el partido completo, para controlar variables relevantes para el estudio del rendimiento (Carling et al., 2008). La utilización de estos sistemas ha servido para confirmar principios básicos del juego que deben ser incluidos en el entrenamiento (Barros et al., 2007). Así el análisis de la competición ha mostrado información relevante respecto a los esfuerzos que acontecen durante el juego, destacando la repetición de acciones de corta duración y alta intensidad, entre las que se intercalan carreras de baja o moderada intensidad a modo de recuperación (Barros et al., 2007; Di Salvo et al., 2007; Mohr, Krustrup \& Bangsbo, 2003; Rampinini, Couts, Castagna, Sassi \& Impellizzeri, 2007; Sales et al., 2014).

La materialización de estas secuencias de esfuerzo y recuperación durante el partido está determinada por elementos relacionados con la propia naturaleza del fútbol (Álvaro et al., 1995) y otros condicionantes externos, que pueden influir en los primeros. A estos condicionantes se los reconoce con el término de variables contextuales (Castellano, Blanco-Villaseñor \& Álvarez, 2011) o situacionales (Lago, Casais, Dominguez \& Sampaio, 2010). Algunas de estas variables hacen referencia a la

Fecha recepción: 21-02-17. Fecha de aceptación: 15-08-17 Javier Sánchez Sanchez

jsanchezsa@upsa.es posición del jugador en el campo (Bradley et al., 2011), el nivel del equipo rival (Hammouda et al., 2013), la categoría competitiva (Bradley etal., 2013), la localización geográfica del partido (Staufenbiel, Riedl \& Strauss, 2016), la temperatura (Chmura, Konefal, Andrzejewski, Kosowski, Rokita \& Chmura, 2016), la altitud (Bohner et al., 2015), el momento del partido (Sparks, Coetzee \& Gabbett, 2016), el resultado momentáneo (Taylor, Mellalieu, James \& Shearer, 2008), el estilo de juego (Tierney, Young, Clarke \& Duncan, 2016), la densidad competitiva (Djaoui et al., 2014), el momento de la temporada (Link \& de Lorenzo, 2016) y el nivel de oposición (Folgado, Duarte, Fernandes \& Sampaio, 2014), entre otros.

Las dimensiones del terreno de juego también pueden afectar al rendimiento condicional de los equipos (Hulka, Weisser \& Belka, 2016). El reglamento FIFA(IFAB, 2016) determina que el área de juego puede variar entre 90-120 m de largo y 45-90 m de ancho. Estas medidas modificarán el área de juego provocando que el espacio de intervención individual del jugador se altere (Parlebás, 2001). Aunque hasta donde conocemos esta variable no ha sido analizada en competición, estudios previos realizados en el contexto de los juegos reducidos han observado que el aumento del tamaño del campo genera un incremento de las distancias cubiertas por los jugadores, especialmente en la carrera a alta velocidad (Casamichana \& Castellano, 2011a; Hodgson, Akenhead \& Thomas, 2014).

Por otra parte, el modelo de competición actual en fútbol profesional se caracteriza por una alta heterogeneidad en los horarios de celebración de los partidos (Hammouda et al., 2013). Esta situación puede alterar el ritmo circadiano o cronotipo de los jugadores (Rae, Stephenson \& Roden, 2015). Aunque esta variable y su influencia sobre la capacidad de rendimiento físico ha sido estudiada desde hace casi un siglo (Pitt, Wolfson \& Moss, 2014), hasta donde conocemos no existen demasiados estudios que se hayan ocupado de analizar la influencia del horario del partido sobre factores fisiológicos relevantes para el rendimiento en el fútbol (Drust, Ahmed \& Roky, 2012). 
Por todo lo anterior, el objetivo del presente estudio ha sido analizar la influencia de las variables situacionales tamaño del campo y horario del partido sobre la distancia total recorrida por partido y los metros completados en diferentes rangos de velocidad de equipos de la Liga de Fútbol Profesional Española.

\section{Método}

\section{Muestra}

22 equipos de la Liga de Fútbol Profesional Española (LFP) que compiten en la Segunda División 'A' fueron analizados durante la temporada 2013/2014. En esta temporada se disputaron 462 partidos oficiales de liga, pero en el estudio se analizaron un total de 317 partidos, lo que representa un $68,61 \%$ de los partidos totales celebrados. Como criterios de exclusión se consideraron: no disponer de la información completa del partido o la expulsión de algún jugador durante el juego. Los datos fueron procesados y analizados manteniendo el anonimato de los participantes. El desarrollo de la investigación se realizó respetando los principios establecidos en la Declaración de Helsinki.

\section{Variables físicas y de situación}

Para el análisis de los metros recorridos se consideróla distancia acumulada por los jugadores participantes en el partido y el resultado se dividió entre 10. También se analizaron los metros recorridos en función de cinco rangos de velocidad establecidos según lo indicado en estudios previos (Barros et al., 2007; Casamichana \& Castellano, 2011b; Di Salvo et al., 2007; Hill-Haas, Dawson, Coutts \& Rowsell, 2009; Rampinini et al., 2007): distancia recorrida a velocidad parado (DPa) $<$ $2 \mathrm{~km} / \mathrm{h}$; distancia recorrida a velocidad caminando (DCa) $2-7.3 \mathrm{~km} / \mathrm{h}$; distancia recorrida a velocidad trotando (DTr) 7.3-14 km/h; distancia recorrida a velocidad corriendo (DCo) $14-21 \mathrm{~km} / \mathrm{h}$; distancia recorrida a velocidad esprintando (DEs) $\geq 21 \mathrm{~km} / \mathrm{h}$.

El análisis de la distancia recorrida y los desplazamientos a diferentes velocidades se realizó en función de las variables independientes tamaño del campo y horario del partido. La información de las dimensiones de los terrenos de juego correspondientes a los equipos participantes, así como del horario de los partidos disputados en la temporada objeto de estudio, fue recogida de la página web oficial de la Real Federación Española de Fútbol. Los campos de juego de los equipos participantes fueron clasificados en dos categorías a partir del cálculo de la mediana (7.140 m): campo grande(CG) y pequeño(CP). Para la distribución de los partidos en función del horario de juego se consideraron tres niveles en función de la hora de finalización del partido: horario del partido por la mañana (PM), tarde (PT) y noche (PN). La descripción de estas variables junto con los registros analizados se recogen en la Tabla 1.

\section{Procedimiento}

Para el registro de los datos de la muestra se ha utilizado el sistema de seguimiento multicámara computarizado TRACAB(Chyronhego@, Estados Unidos), instalado en los estadios. Esta información fue gestionada por Mediacoach (Mediapro®, España), a partir del cual se generaban informes de las demandas físicas de variables predefinidas. Estudios previos han confirmado la fiabilidad y validez de este tipo de sistemas de seguimiento de vídeo para valorar las demandas físicas de los jugadores en competición (Buchheit, Allen, Poon, Modonutti, Gregson \& Di Salvo, 2014; Castellano et al., 2014; Di Salvo, Collins, McNeill \& Cardinale, 2006; Randers et al., 2010). Los informes generados se gestionaron en Microsoft Excel, y a partir de aquí se implementaron las diferentes técnicas analíticas.

\section{Análisis estadístico}

El estudio de las variables mostró una distribución normal de acuerdo a la prueba de Shapiro-Wilk. La comparación de las variables distancia recorrida y desplazamientos a diferentes velocidades entre CG y CP fue realizada con la prueba $t$ student para muestras independientes. El comportamiento de las variables dependientes en función del horario de partido PM, PT y PN se realizó aplicando ANOVA de un factor, con análisis post hoc de Bonferroni. En todos los casos se consideraron diferencias significativas cuando $\mathrm{p}<0,05$. Además para calcular la magnitud del efecto asociada a la relación entre variables dependientes, se aplicó el tamaño del efecto (TE). Los valores cuantitativos fueron establecidos a partir de la d de Cohen considerando: $<0,19$ (leve); 0,2-0,59 (pequeño); 0,6-1,19 (moderado); 1,2-2 (grande); >2 (muy grande) (Hopkins, 2000). El análisis de los datos fue realizado con el paquete estadístico para las ciencias sociales SPSS 19.0 (SPSS Inc., USA).

\section{Resultados}

Respecto a la influencia del tamaño del campo sobre el comportamiento físico de los equipos, los resultados indican que la distancia recorrida es significativamente mayor $(\mathrm{p}<0,05$; $\mathrm{TE}=0,32)$ en CG que CP (11.905 $\pm 463 \mathrm{~m}$ vs $11.722 \pm 631 \mathrm{~m}$, respectivamente). En el análisis de la distancia recorrida en diferentes rangos de velocidad (Figura 1), DPa y DCo son significativamente mayores ( $<<0,01$ ) en CG que en CP (521 $\pm 288 \mathrm{~m}$ y $2.241 \pm 219 \mathrm{~m}$ vs $402 \pm 344 \mathrm{~m}$ y $2.165 \pm 222$ $\mathrm{m}$, respectivamente). En CP la distancia DCa $(4.177 \pm 234 \mathrm{~m})$ es significativamente mayor $(\mathrm{p}<0,05 ; \mathrm{TE}=0,23)$ que en CG $(4.126 \pm 194$ $\mathrm{m})$.

No existen diferencias significativas ( $\mathrm{p}>0,05)$ en la distancia recorrida por partido en función de la hora a la que se disputa la competición $(\mathrm{PM}=11.609 \pm 586 \mathrm{~m} ; \mathrm{PT}=11.790 \pm 591 \mathrm{~m} ; \mathrm{PN}=11.843 \pm 569 \mathrm{~m})$. Tal y como refleja la Figura 2, el estudio de los desplazamientos tampoco muestra diferencias en ninguna de las variables salvo en DCo, donde se observa menores valores $(\mathrm{p}<0,05)$ en $\mathrm{PM}(2.111 \pm 197 \mathrm{~m})$ que en PT $(2.189 \pm 244 \mathrm{~m})$ y PN $(2.262 \pm 218 \mathrm{~m})$.

\begin{tabular}{|c|c|c|}
\hline Variable & Definición & Registros \\
\hline CG & $\geq 7.140 \mathrm{~m}$ & 443 \\
\hline $\mathrm{CP}$ & $<7.140 \mathrm{~m}$ & 192 \\
\hline PM & $<14: 30 \mathrm{~h}$ & 59 \\
\hline PT & $14: 30$ a $20: 00 \mathrm{~h}$ & 527 \\
\hline PN & $<20: 00 \mathrm{~h}$ & 49 \\
\hline
\end{tabular}

por la tarde; $\mathrm{PN}=$ partido por la noche.

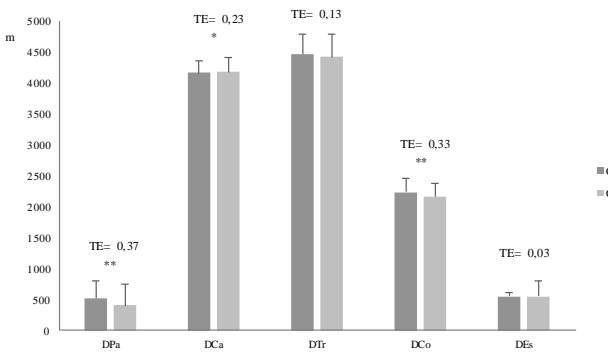

Figura 1. Distancia cubierta en 5 rangos de velocidad según el tamaño del campo de juego. Nota: $\mathrm{TE}=$ Tamaño del Efecto; $\mathrm{CG}=$ Campo Grande; $\mathrm{CP}$ = Campo Pequeño; $\mathrm{DPa}=$ Distancia recorrida en velocidad $<2 \mathrm{~km} / \mathrm{h}$; DCa = Distancia recorrida en velocidad 2-7,3 km/h; DTr = Distancia recorrida en velocidad 7,3-14 km/h; DCo = Distancia recorrida en velocidad 14-21 $\mathrm{km} / \mathrm{h}$; DEs $=$ Distancia recorrida en velocidad $\geq 21 \mathrm{~km} / \mathrm{h}$.

*,** Indica diferencias significativas entre CG y CP ( $\mathrm{p}<0,05$ y $\mathrm{p}<0,01$, respectivamente)

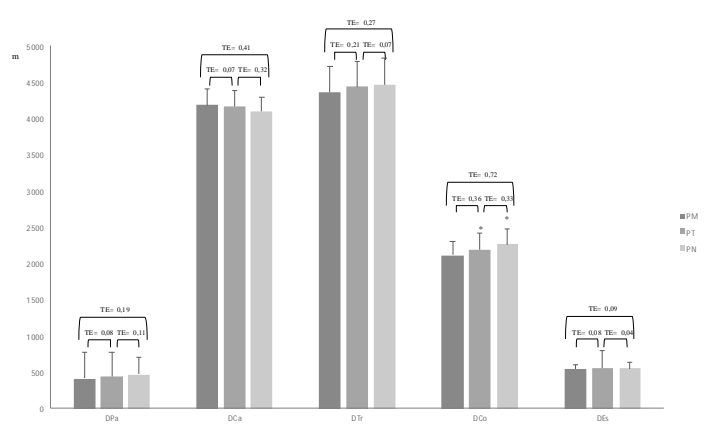

Figura 2. Distancia cubierta en 5 rangos de velocidad en función del horario del partido. Nota: TE = Tamaño del Efecto; PM = Partido en horario de mañana; $\mathrm{PT}$ = Partido en horario de tarde; PN = Partido en horario de noche; DPa = Distancia recorrida en velocidad $<2 \mathrm{~km} / \mathrm{h}$; DCa = Distancia recorrida en velocidad 2-7,3 km/h; DTr = Distancia recorrida en velocidad 7,3-14 $\mathrm{km} / \mathrm{h}$; DCo $=$ Distancia recorrida en velocidad $14-21 \mathrm{~km} / \mathrm{h}$; DEs $=$ Distancia recorrida en velocidad $\geq 21 \mathrm{~km} / \mathrm{h}$.

* Indica diferencias significativas con PM $(p<0,05)$ 


\section{Discusión}

El objetivo del presente estudio fue analizar la influencia de las variables situacionales tamaño del campo y horario del partido sobre la distancia total recorrida por partido y la completada en diferentes rangos de velocidad en equipos de la Liga de Fútbol Profesional Española. Los resultados mostraron que la distancia completada por partido en los rangos DPa y DCo era mayor cuando se jugaba en CG respecto a CP. La variable horario del partido parece que no influyó sobre el rendimiento físico de los equipos, encontrándose menos distancia recorrida en DCo cuando PM respecto a PT y PN.

Los resultados del estudio mostraron que los equipos recorrieron más metros cuando jugaban en CG. Hasta donde conocemos no existen trabajos que hayan analizado la influencia de esta variable sobre las demandas físicas de jugadores profesionales en situación de partido oficial. Sin embargo estudios previos que analizaron la carga asociada a los juegos reducidos coincidieron en señalar que un aumento en la superficie de juego y, por tanto, un mayor espacio de interacción individual (Casamichana \& Castellano, 2010), tiene como consecuencia una mayor distancia recorrida por los jugadores (Hulka et al., 2016). Esta modificación del espacio relativo por jugador que se da en CG respecto al CP implica que el jugador tenga que abarcar más superficie de juego, lo que se refleja en el aumento de la distancia total recorrida (Hill-Haas, Dawson, Impellizzeri \& Coutts, 2011). Este aspecto debería tenerse en cuenta por los entrenadores a la hora de diseñar el plan estratégico prepartido. En la práctica debería materializarse en la gestión del número de jugadores y del espacio de juego correspondiente a las tareas de entrenamiento, y todo ello sin perder la esencia de los comportamientos técnico-tácticos que quieran reproducirse.

Respecto a la distancia acumulada en diferentes rangos de velocidad, se observó que el CG respecto al CP provocaba el aumento de los metros en DPa y DCo y la disminución de la distancia recorrida en DCa. El estudio de la carga asociada a los juegos reducidos ha indicado que los desplazamientos en baja, media y alta velocidad también eran mayores cuando el espacio relativo por jugador se incrementaba en la tarea (Casamichana \& Castellano, 2010). Es posible que a mayor espacio se disminuya la densidad de acciones del jugador, debido a que puede estar más tiempo lejos del foco de juego y esto incluir la ejecución de más desplazamientos a baja velocidad (Silva, Garganta, Santos \& Teoldo, 2014). Al contrario de lo señalado en trabajos previos (Casamichana \& Castellano, 2010), en nuestro estudio no hemos podido observar cambios en los desplazamientos a alta velocidad como consecuencia de la modificación de las dimensiones del espacio de juego. Quizás esto pueda deberse a que en todos los campos de los equipos analizados las dimensiones son grandes comparados con lo que habitualmente se implementa en el estudio de los juegos reducidos (Hill-Haas et al., 2011). Por otro lado, se ha observado que reducir el espacio de juego llevaba consigo la modificación del comportamiento táctico de los equipos, debido a que los jugadores ocupaban situaciones más cercanas entre sí (Frencken, Van Der Plaats, Visscher \& Lemmink, 2013). Esta mayor proximidad limitó el espacio para realizar movimientos a alta velocidad, pero aumentó la demanda neuromuscular de la tarea (Castellano \& Casamichana, 2013).

En el presente trabajo únicamente se observaron valores mayores en DCo cuando el partido se jugaba en PT y PN respecto a PM. Otros estudios que analizaron el rendimiento en fútbol australiano empleando umbrales de velocidad iguales a los de este trabajo, no encontraron diferencias en indicadores de alta intensidad, y únicamente observaron un aumento de la distancia recorrida por partido cuando se jugaba en horario de noche (Ryan et al., 2015). Aunque parece que los ritmos circadianos y con ello la predisposición hacia el rendimiento se incrementaba progresivamente desde el principio hasta el final del día (Dvorak \& Racinais, 2010), también se ha observado que la respuesta física del futbolista empeoraba hacia las últimas horas (Chtourou, Hammouda, Souissi, Chamari, Chaouachi \& Souissi, 2012; Reilly, Atkinson, Edwards, Waterhouse, Farrelly \& Fairhurst, 2007; Ryan et al., 2015). Las variaciones en el rendimiento podrían ser debidas a las modificaciones en la temperatura corporal ocurridas a lo largo del ciclo diurno (Borms \& Van Roy, 2001), junto a cambios en la predisposición mental hacia el esfuerzo ocurrida con el paso de las horas (Hammouda et al., 2013). Con respecto a la temperatura corporal, parece que el período que va entre las 16 y 20 horas podría reunir las mejores condiciones para proyectar el rendimiento de los deportistas (Reilly et al., 2007). Sin embargo también existen estudios que no pudieron confirmar cambios en diferentes capacidades físicas a través de test de campo realizados en diferentes momentos del día (Souza, Carvalho, Rodrigues, Cymrot, Saeta \& Blascovi-Assis, 2015; Rae et al., 2015).

A pesar de los resultados obtenidos, una de las limitaciones del estudio ha sido el empleo de la mediana para categorizar los campos de juego en función de sus dimensiones. El empleo de esta técnica ubica campos de dimensiones muy similares en categorías diferentes. En futuros estudios debe valorarse el empleo de la técnica cluster de clasificación de variables, ya que en el presente trabajo su uso habría reducido la muestra en alguna de las categorías perdiendo consistencia en el análisis estadístico. Respecto a la hora del partido, no fue posible controlar las estrategias de aclimatación al horario desarrolladas por los equipos, lo que podría haber influido en los resultados obtenidos. Además, para el análisis de las demandas físicas asociadas a las variables situaciones estudiadas, no se tuvieron en cuenta las demarcaciones de los jugadores. El puesto ocupado en el campo determina el comportamiento físico del jugador (Di Salvo et al., 2007), por lo que sería interesante analizar el comportamiento de las variables situacionales en consonancia con la propia especificidad del esfuerzo del futbolista. Finalmente, el hecho de haber podido disponer de otras variables de análisis de la carga interna como el esfuerzo percibido o la frecuencia cardíaca podrían haber aportado una visión más completa de la demanda de competición en relación a las variables contextuales.

\section{Conclusiones}

El estudio de las variables situacionales es clave para comprender la demanda condicional de los equipos en competición. Aunque en los últimos años este ámbito de estudio ha sido ampliamente atendido, existen algunas variables poco estudiadas en equipos de alto nivel. Los resultados de nuestro trabajo revelan que en una muestra de futbolistas de categoría profesional, la dimensión del terreno de juego condiciona la demanda física debido a que en un campo grande se incrementan los metros recorridos y se modifica la distancia recorrida en los diferentes rangos de velocidad respecto a un campo pequeño. Sin embargo, no existe una influencia significativa del horario de partido sobre la mayoría de variables analizadas para determinar la respuesta física de los equipos en competición.

Estos resultados deben ser valorados por entrenadores y preparadores físicos para entender las demandas físicas del fútbol y poder emplear estrategias de intervención durante los microciclos de competición. Además, es útil para mejorar las estrategias de preparación prepartido y también para reforzar los protocolos de recuperación postcompetición.

\section{Referencias}

Álvaro, J., Dorado, A., González, J., Navarro, F, Molina, J., Portolés, J., \& Sánchez, F. (1995). Modelo de análisis de los deportes colectivos basado en el rendimiento en competición. INFOCOES, 1(0), 21-40.

Barros, R. M., Misuta, M. S., Menezes, R. P., Figueroa, P. J., Moura, F. A., Cunha, S., Anido, R., \& Leite, N. J. (2007). Analysis of the distances covered by first division Brazilian soccer players obtained with an automatic tracking method. Journal of Sports Science y Medicine, 6(2), 233-242.

Bohner, J. D., Hoffman, J. R., McCormack, W. P., Scanlon, T. C., Townsend, J. R., Stout, J. R., Fragala, M.S., \& Fukuda, D. H. (2015). Moderate Altitude Affects High Intensity Running Performance in a Collegiate Women's Soccer Game. Journal of Human Kinetics, 47(1), 147154.

Borms, J., \& Van Roy, P. (2001). Flexibility. In Eston, R. \& Reilly, T. (Eds), Kinanthropometry and exercise physiology laboratory manual: tests, procedures, Vol. I (pp. 117-147). London: Routledge.

Bradley, P., Carling, C., Archer, D., Roberts, J., Dodds, A., Di Mascio, M., 
Darren, P., Gomez-Diaz, A., Peart, D., \& Krustrup, P. (2011). The effect of playing formation on high-intensity running and technical profiles in English FA Premier League soccer matches. Journal of Sports Sciences, 29(8), 821-830.

Bradley, P., Carling, C., Gómez-Diaz, A., Hood, P., Barnes, C., Ade, J., Boddy, M., Krustrup, P., \& Mohr, M. (2013). Match performance and physical capacity of players in the top three competitive standards of English professional soccer. Human Movement Science, 32(4), 808-821.

Buchheit, M., Allen, A., Poon, T., Modonutti, M., Gregson, W., \& Di Salvo, V. (2014). Integrating different tracking systems in football: multiple camera semi-automatic system, local position measurement and GPS technologies. Journal of Sports Sciences, 32(20), 1844 1857.

Carling, C., Bloomfield, J., Nelsen, L., \& Reilly, T. (2008). The role of motion analysis in elite soccer. Contemporary performance measurement techniques and work rate data. Sport Medicine, 38(10), 839-862.

Casamichana, D., \& Castellano, J. (2010). Time motion, heart rate, perceptual and motor behaviour demands in small-sides games: Effects of pitch size. Journal of Sports Sciences, 28(4), 1615-1623.

Casamichana, D., \& Castellano, J. (2011a). Demanda fisiológica en juegos reducidos en fútbol con diferente orientación del espacio. International Journal of Sport Science, VII (23), 141-154.

Casamichana, D., \& Castellano, J. (2011b). Demandas físicas en jugadores semiprofesionales de fútbol: ¿se entrena igual que se compite? Cultura, Ciencia y Deporte, 7(17), 121-127.

Castellano, J., Álvarez-Pastor, D., \& Bradley, P. (2014). Evaluation of research using computerised tracking systems (amisco ${ }^{\circledR}$ and prozone $\left.{ }^{\circledR}\right)$ to analyse physical performance in elite soccer: A systematic review. Sports Medicine, 44, 701-712.

Castellano, J., Blanco-Villaseñor, A., \& Álvarez, D. (2011). Contextual Variables and Time-Motion Analysis in Soccer. International Journal of Sports Medicine, 32(6), 415-421.

Castellano, J., \& Casamichana, D. (2013). Differences in the number of accelerations between small-sided games and friendly matches in soccer. Journal of Sports Science and Medicine, 12(1), 209-210.

Chmura, P., Konefal, M., Andrzejewski, M., Kosowski, J., Rokita, A., \& Chmura, J. (2016). Physical activity profile of 2014 FIFA World Cup players, with regard to different ranges of air temperature and relative humidity. International Journal of Biometeorolgy, 1-8.

Chtourou, H., Hammouda, O., Souissi, H., Chamari, K., Chaouachi, A., \& Souissi, N. (2012). Diurnal Variations in Physical Performances Related to Football in Young Soccer Players. Asian Journal of Sports Medicine, 3(3), 139-144.

Di Salvo, V., Baron, R., Tschan, H., Calderón-Montero, F. J., Bachl, N., \& Pigozzi, F. (2007). Performance characteristics according to playing position in elite soccer. International Journal of Sports Medicine, 28(3), 222-7.

Di Salvo, V., Collins, A., McNeill, B., \& Cardinale, M. (2006). Validation of Prozone $\mathbb{~}$ : A new video-based performance analysis system. International Journal of Performance Analysis in Sport, 6(1), 1-1.

Djaoui, L., Wong, D., Pialoux, V., Hautier, C., Da Silva, C., Chamari, K., \& Dellal, A. (2014). Physical Activity during a Prolonged Congested Period in a Top-Class European Football Team, 5(1), 47-53.

Drust, B., Ahmed, Q., \& Roky, R. (2012). Circadian variation and soccer performance: Implications for training and match-play during Ramadan. Journal of Sports Sciences, 30(1), 43-52.

Dvorak, J., \& Racinais, S. (2010). Training and playing football in hot environments. Scandinavian Journal of Medicine \& Science in Sports, 20(3), 4-5.

Folgado, H., Duarte, R., Fernandes, O., \& Sampaio, J. (2014). Competing with Lower Level Opponents Decreases Intra-Team Movement Synchronization and Time-Motion Demands during Pre-Season Soccer Matches. PLoS ONE, 9(5), e97145.

Frencken, W., Van Der Plaats, J., Visscher, C., \& Lemmink, K. (2013). Size matters: Pitch dimensions constrain interactive team behaviour in soccer. Journal of Systems Science and Complexity, 26(1), 85-93.

Hammouda, O., Chtourou, H., Aloui, A., Chahed, H., Kallel, C., Miled, A. ... \& Souissi, N. (2013). Concomitant Effects of Ramadan Fasting and Time-Of-Day on Apolipoprotein AI, B, Lp-a and Homocysteine Responses during Aerobic Exercise in Tunisian Soccer Players. PLoS ONE, 8(11), e79873.

Hammouda, O., Chtourou, H., Chaouachi, A., Chahed, H., Bellimem, H., Chamari, K., \& Souissi, N. (2013). Time-of-day effects on biochemical responses to soccer-specific endurance in elite Tunisian football players. Journal of Sports Sciences, 31(9), 963-971.
Hill-Haas, S. V., Dawson, B. T., Coutts, A. J., \& Rowsell, G. J. (2009). Physiological responses and time-motion characteristics of various small-sided soccer games in youth players. Journal of Sports Sciences, 27(1), 1-8.

Hill-Haas, S. V., Dawson, B., Impellizzeri, F. M., \& Coutts, A. J. (2011). Physiology of small-sided games training in football: a systematic review. Sports Medicine, 41(3), 199-220.

Hodgson, C., Akenhead, R., \& Thomas, K. (2014). Time-motion analysis of acceleration demands of $4 \mathrm{v} 4$ small-sided soccer games played on different pitch sizes. Human Movement Science, 33, 25-32.

Hopkins, W. G. (2000). Measures of Reliability in Sports Medicine and Science. Sports Medicine, 30(1), 1-15.

Hulka, K., Weisser, R., \& Belka, J. (2016). Effect of the Pitch Size and Presence of Goalkeepers on the Work Load of Players During SmallSided Soccer Games. Journal of Human Kinetics, 51, 175-181.

IFAB. (2016). Reglas del Juego 2016/2017. Zúrich: FIFA.

Lago, C., Casais, L., Dominguez, E., \& Sampaio, J. (2010). The effects of situational variables on distance covered at various speeds in elite soccer. European Journal of Sport Science, 10(2), 103-109.

Link, D., \& de Lorenzo, M. (2016). Seasonal Pacing - Match Importance Affects Activity in Professional Soccer. PLoS ONE, 11(6), e0157127.

Mohr, M., Krustrup, P., \& Bangsbo, J. (2003). Match performance of high-standard soccer players with special reference to development of fatigue. Journal of Sports Sciences, 21(7), 519.

Parlebás, P. (2001). Juegos, deporte y sociedad. Léxico de praxiología motriz. Barcelona: Paidotribo.

Pitt, T., Wolfson, S., \& Moss, M. (2014). The relationship between fear of failure and self-talk in winning and losing situations. Sport and Exercise Psychology Review, 10(1), 91-95.

Rae, D., Stephenson, K., \& Roden, L. (2015). Factors to consider when assessing diurnal variation in sports performance: the influence of chronotype and habitual training time-of-day. European Journal of Applied Physiology, 115(6), 1339-1349.

Rampinini, E., Couts, A., Castagna, C., Sassi, R., \& Impellizzeri, F. M. (2007). Variation in Top Level Soccer Match Performance. International Journal of Sports Medicine, 28(12), 1018-1024.

Randers, M., Mujika, I., Hewitt, A., Santisteban, J., Bischoff, R., Solano, R., Zubillaga, A., Peltola, E., Krustrup, P., \& Mohr, M. (2010). Application of four different football match analysis systems: A comparative study. Journal of Sports Sciences, 28(2), 171-182.

Reilly, T., Atkinson, G., Edwards, B., Waterhouse, J., Farrelly, K., \& Fairhurst, E. (2007). Diurnal Variation in Temperature, Mental and Physical Performance, and Tasks Specifically Related to Football (Soccer). Chronobiology International: The Journal of Biological \& Medical Rhythm Research, 24(3), 507-519.

Ryan, R., Bahnert, A., McBrien, J., Siegler, J., \& Lovell, R. (2015). The effect of chronotype upon physical performance during Australian rules football matches scheduled in the morning, afternoon and evening. Journal of Australian Strength and Conditioning, 23(6), 101-104.

Sales, M. M., Browne, R. A., Asano, R. Y., dos Reis Vieira Olher, R., Novad, J. F., \& Simões, H. G. (2014). Physical fitness and anthropometric characteristics in professional soccer players of the United Arab Emirates. Revista Andaluza de Medicina Del Deporte, 7(3), 106-110.

Silva, B., Garganta, J., Santos, R., \& Teoldo, I. (2014). Comparing Tactical Behaviour of Soccer Players in 3 vs. 3 and 6 vs. 6 small sided games. Journal of Human Kinetics, 41, 191-202.

Souza, P. R., Carvalho, S. G., Rodrigues, G. M., Cymrot, R., Saeta, B. R., \& Blascovi-Assis, S. M. (2015). Motor coordination and circadian rhythm in boys with different development characteristics. Revista Neurociências, 23(3), 342-348.

Sparks, M., Coetzee, B., \& Gabbett, T. J. (2016). Variations in highintensity running and fatigue during semi-professional soccer matches. International Journal of Performance Analysis in Sport, 16(1), 122132.

Staufenbiel, K., Riedl, D., \& Strauss, B. (2016). Learning to be advantaged: The development of home advantage in high-level youth soccer. International Journal of Sport and Exercise Psychology, 1-15.

Taylor, J. B., Mellalieu, S. D., James, N., \& Shearer, D. A. (2008). The influence of match location, quality of opposition, and match status on technical performance in professional association football. Journal of Sports Sciences, 26(9), 885-895.

Tierney, P. J., Young, A., Clarke, N. D., \& Duncan, M. J. (2016). Match play demands of 11 versus 11 professional football using Global Positioning System tracking: Variations across common playing formations. Human Movement Science, 49, 1-8. 